\title{
Anne Clerval, 2013, Paris sans le peuple, Paris, La Découverte, $254 \mathrm{p}$.
}

Pierre-Alexis Tchernoïvanoff

\section{(2) OpenEdition}

\section{Journals}

Electronic version

URL: http://journals.openedition.org/ress/2756

DOI: 10.4000/ress.2756

ISSN: $1663-4446$

\section{Publisher}

Librairie Droz

\section{Printed version}

Date of publication: 6 May 2014

Number of pages: $294-296$

ISBN: 978-2-600-01829-6

ISSN: 0048-8046

Electronic reference

Pierre-Alexis Tchernoïvanoff, « Anne Clerval, 2013, Paris sans le peuple, Paris, La Découverte, 254 p. », Revue européenne des sciences sociales [Online], 52-1 | 2014, Online since 12 May 2014, connection on 22 September 2020. URL : http://journals.openedition.org/ress/2756 ; DOI : https://doi.org/10.4000/ ress. 2756

This text was automatically generated on 22 September 2020

(c) Librairie Droz 


\title{
Anne Clerval, 2013, Paris sans le peuple, Paris, La Découverte, 254 p.
}

\author{
Pierre-Alexis Tchernoïvanoff
}

1 Tiré d'une thèse de doctorat, l'ouvrage d'Anne Clerval éclaire les évolutions récentes de Paris à la lumière d'un concept hérité de la géographie radicale anglo-saxonne, celui de gentrification. L'auteure, géographe de formation et enseignante-chercheuse à l'université Paris-Est Marne-la-Vallée, fonde son étude sur deux démarches complémentaires. D'une part, une approche statistique construite à partir des données du recensement de la population parisienne; d'autre part, sur des enquêtes de terrain menées entre 2004 et 2007 dans trois quartiers de Paris révélateurs de « différents stades de progression de la gentrification » (p.11). Outre des observations régulières, l'auteure a réalisé quatre-vingt entretiens semi-directifs et traité trente et un questionnaires envoyés à une centaine de foyers. Les personnes enquêtées rassemblent à la fois des acteurs publics et privés, de nationalité française ou non, des habitants issus des classes populaires et d'autres, plus aisés, nouveaux arrivants dans les quartiers concernés. Alliant dimension historique, sociogéographique et politique, l'ouvrage a pour objectif d'éclairer un processus relativement peu investi par la littérature scientifique française. L'introduction est pour l'auteure l'occasion de préciser le cadre théorique qui est le sien. En rapprochant les évolutions du système capitaliste mondial et l'éviction progressive des catégories populaires des centresvilles, le concept de gentrification "s'inscrit dans une interaction dialectique entre l'espace urbain et l'espace social». Il faut donc l'envisager comme «le processus par lequel l'espace urbain central est adapté à l'état actuel des rapports sociaux » (ibid.).

2 La première partie de l'ouvrage est consacrée à une relecture de l'histoire de la capitale à la lumière du concept de gentrification. En montrant bien l'importance de la rénovation haussmannienne dans l'enrichissement de Paris, l'auteure insiste sur les conséquences de la désindustrialisation dans l'embourgeoisement de la ville. À partir des années 1960, cet embourgeoisement a progressivement pénétré les quartiers traditionnellement populaires. La tertiarisation de l'économie, reléguant l'activité ouvrière dans des espaces périurbains, a eu pour effet le remplacement progressif des 
classes populaires par une petite bourgeoisie intellectuelle, composée de cadres, de professionnels de l'information et du spectacle, ainsi que d'étudiants. Anne Clerval souligne bien le caractère tardif du processus de gentrification à Paris. Comparativement aux autres grandes métropoles anglaises et nord-américaines, la gentrification parisienne ne s'opère et ne devient qu'ostensible à partir de la fin des années 1990. Le contrôle des loyers par la loi de 1948 et l'indexation des loyers sur la surface et la qualité des logements, et non sur les prix du marché, a en effet freiné la spéculation immobilière, et ce jusqu'aux années 1980. Cela explique également la relative faiblesse des loyers par rapport à d'autres métropoles comme New York ou Londres. D'autre part, les politiques de rénovation menées par la municipalité de droite dans les années 1980-1990 ont eu un effet ambigu : en dépit de leur objectif d'élever la capitale au rang de métropole et de niveler par le haut le niveau social de la population, elles ont malgré tout conduit à la construction d'un parc conséquent de logements sociaux, assurant ainsi le maintien intra-muros d'une partie des classes populaires.

3 La seconde partie de l'ouvrage se centre sur la géographie du processus de gentrification. Sont étudiées « les modalités particulières de la gentrification au niveau local, tant dans l'habitat que dans les commerces et la fréquentation de l'espace public» (p. 63). Les données du recensement et une analyse à l'échelle des IRIS [Ilots Regroupés pour l'Information Statistique. Un IRIS regroupe environ 2000 habitants et constitue le découpage géographique le plus fin proposé par l'Insee] permettent à Anne Clerval de situer avec précision le processus de gentrification dans le temps et dans l'espace. À partir d'un rappel historique de la division sociale de la capitale, scindée entre un Sud et Ouest bourgeois d'un côté, et un Nord et Est populaires de l'autre, l'auteure analyse la construction d'un front de gentrification évoluant vers le Nord et l'Est de la ville. Le processus de gentrification, au-delà des spécificités des quartiers gentrifiés, obéit à des procédés similaires. Il s'appuie sur la construction de logements neufs et sur la réhabilitation des logements anciens puis sur l'appropriation de ses quartiers populaires par les gentrifieurs. Initiée par les artistes et les intellectuels précaires, cette vague est ensuite amplifiée par, d'une part, les promoteurs immobiliers, puis, d'autre part, par les commerçants du quartier qui, voyant arriver des habitants séduits par l'imaginaire cosmopolite et populaire, adaptent leurs prix à des nouveaux arrivants plus fortunés. Cette première arrivée ouvre la voie à une seconde vague de ménages gentrifieurs plus fortunés, majoritairement composés de cadres du privé, d'ingénieurs et de professionnels des métiers de la culture.

4 Au travers des enquêtes menées par l'auteure dans les quartiers Saint-Antoine, du Faubourg du Temple et de Château Rouge, la troisième et dernière partie de l'ouvrage traite des rapports sociaux de domination qui s'y configurent. Le groupe des gentrifieurs, que l'auteure qualifie de "petite bourgeoisie nouvelle», adopte un comportement ambigu vis-à-vis des classes populaires vivant déjà sur place : valorisant le concept de mixité sociale, elle adopte en réalité des pratiques favorisant l'entre-soi et l'évitement des écoles publiques du quartier. Centrant son analyse sur l'action municipale depuis l'arrivée de la gauche en 2001, Anne Clerval souligne les limites de la construction de logements sociaux et celles du discours valorisant la mixité sociale, qu'elle juge flou et ambigu. Le volontarisme de la municipalité en la matière n'a ni permis de combler les besoins, ni de compenser les destructions. Concentrée dans le logement intermédiaire, cette politique a de surcroît davantage profité aux classes moyennes qu'aux classes populaires. La municipalité, par sa "politique volontaire d'embellissement de la ville et d'amélioration du cadre de vie » (p. 183), s'est également 
rendue complice du processus de gentrification par le soutien à la création de lieux culturels fréquentés majoritairement par les gentrifieurs de la ville. L'auteure tire un constat relativement pessimiste quant aux remparts à ce processus de gentrification. En l'absence de volonté politique, la seule résistance s'organise par l'entremise des communautés fortement structurées, seules capables de venir concurrencer les gentrifieurs dans l'acquisition de logements et de commerces.

5 Au terme de la lecture de l'ouvrage, on se permettra une remarque sur la généralité du phénomène analysé par l'auteure. Se dégage en effet le tableau d'une ville dont aurait été éradiquée la pauvreté de ses habitants, par leur remplacement et leur éviction. Embourgeoisée jusqu'en ses bordures, la capitale ne connaîtrait plus la pauvreté qu'en ses marges les plus réduites. Certaines enquêtes nuancent cependant un tel propos. Une étude récente menée par le Centre d'observation et de mesure des politiques d'action sociale (Compas) sur la pauvreté dans les territoires met ainsi en lumière la persistance de niveaux de pauvreté importants dans certains arrondissements du Nord et de l'Est de la capitale. Il en ressort que les $18 \mathrm{e}, 19$ e et 20 e arrondissements ont des taux de pauvreté supérieurs à $20 \%$, dépassant ainsi assez largement celui de la moyenne nationale, dont le taux s'élève aujourd'hui à $14 \%$. Des poches importantes de pauvreté demeurent, même si l'élévation globale du niveau de vie des habitants de ses quartiers n'est pas remise en cause. Cet apport ne remet en cause ni la démarche ni la profondeur du propos d'Anne Clerval. Il apporte peut-être quelques précisions à un ouvrage dont l'engagement tend parfois à détourner le lecteur de toute sa subtilité et de ses nuances.

\section{AUTHORS}

\section{PIERRE-ALEXIS TCHERNOÏVANOFF}

Université Paris Descartes - GEPECS 